\title{
Encoding CNN Activations for Writer Recognition
}

\author{
Vincent Christlein, Andreas Maier \\ Pattern Recognition Lab, Friedrich-Alexander-Universität Erlangen-Nürnberg, 91058 Erlangen, Germany \\ vincent.christlein@fau.de, andreas.maier@fau.de
}

\begin{abstract}
The encoding of local features is an essential part for writer identification and writer retrieval. While CNN activations have already been used as local features in related works, the encoding of these features has attracted little attention so far. In this work, we compare the established VLAD encoding with triangulation embedding. We further investigate generalized max pooling as an alternative to sum pooling and the impact of decorrelation and Exemplar SVMs. With these techniques, we set new standards on two publicly available datasets (ICDAR13, KHATT).
\end{abstract}

Index Terms-writer identification; writer retrieval; deep learning; document analysis

\section{INTRODUCTION}

Handwritings play an important role for law enforcement agencies in proving someone's authenticity because it can be used as a biometric identifier like faces or speech. Forensic experts are consulted to make a decision in such scenarios. However, searching for a particular writer in a huge dataset requires automatic or semi-automatic methods. Due to the mass-digitization processes of historical documents, this topic has also attracted attention in the field of historical document analysis [1]-[3].

In this work, we focus on the task of offline writer recognition, in particular writer identification and writer retrieval. Writer identification denotes the problem of finding the writer of a query handwriting in a dataset of known writers. For writer retrieval, all handwritings of a dataset are ranked according to their similarity to the query sample.

Writer identification/retrieval methods can be grouped into codebook-based methods and codebook-free methods. Codebook-based methods rely on a codebook that serves as background model. This model is used to compute statistics that form the global descriptor which is compared with each other, a process known as encoding. Codebook-free methods compute a global image descriptor directly from the handwriting. For example, the width of the ink trace [1] or the angles of stroke directions [4] were used for writer identification purposes.

We employ features extracted from a deep convolutional neural network (CNN). CNNs are the state-of-the-art tool for image classification since the AlexNet CNN [5] won the ImageNet competition. They also became more and more popular in the domain of document analysis [6]-[8] and recently also in the field of writer recognition [9]-[12].

In this work, we investigate multiple parts of a codebookbased writer identification/retrieval pipeline using features computed by means of a CNN. We try to answer the following questions: 1) Produces a deeper network better activations? 2) Which encoding method performs better? More specifically, we compare vectors of locally aggregated descriptors (VLAD) [13] with the more recent triangulation embedding [14], which showed superior performance to other encoding methods using traditional features [15] and CNN activation features [16] 3) Another recent improvement concerns the aggregation of local descriptors. Does generalized max pooling (GMP) [17] work better than sum pooling? 4) Which effects do PCA whitening on CNN activations have and how much impact do Exemplar SVMs have?

The paper is organized as follows: After reporting about related work in Sec. II, the general pipeline, the encoding methods and generalized max pooling are outlined in Sec. III. In Sec. IV, the different parts of our pipeline are evaluated before the paper is concluded in Sec. V.

\section{RELATED WORK}

One of the first writer recognition methods using deep learning techniques was introduced by Fiel and Sablatnig [9]. They proposed the use of convolutional neural network (CNN) activations obtained from the penultimate layer of a trained CNN. The eight layer deep CNN was trained by word or line segmentations obtained from the IAM dataset. The mean of the CNN activation features were used as feature vector which were compared using the $\chi^{2}$-distance. They improved upon the state of the art (at that time) on the IAM and the ICFHR'12 datasets. At the same time, they show a worse result on the ICDAR13 dataset (containing Greek and English samples), which can be attributed to wrong word segmentations and the missing Greek training data.

Instead, we proposed to encode the CNN activation features by means of GMM supervectors [10], which are subsequently compared by the cosine distance. We showed improved mAP on the ICDAR13, CVL and KHATT dataset.

In contrast to these works [9], [10], Xing and Quiao [11] proposed the use of a network structure consisting of two branches sharing the convolutional layers. Two adjacent image patches of the same writer, i. e., two non-overlapping windows of an input line, are used as input for the network. The authors report good performance results on the IAM dataset. However, the $\mathrm{CNN}$ is trained for a specific writer on a line-basis using some lines for training, one for validation and one for testing. In other words, they used an end-to-end training for the writers which makes a comparison with other retrieval/identificationbased publications impossible.

Tang and $\mathrm{Wu}$ [12] considered the global appearance as an important feature. They do not employ a patch-wise approach but use full document images which are created artificially by 


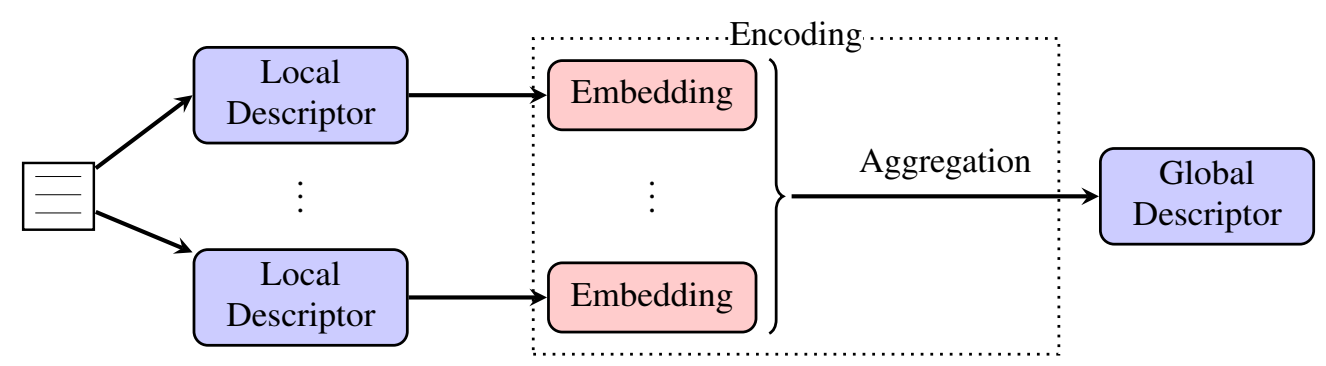

Fig. 1: Encoding of local descriptors to form a global representation which can be compared.

means of random segmented words from the original writing. In this way, they generated about 500 training and about twenty testing samples per writer to get more diverse feature vectors. The similarity is computed by means of the log-likelihood ratio of the CNN activation features of the penultimate layer. They presented the current best results on the CVL dataset and the second best results using the ICDAR13 dataset.

\section{Methodology}

Commonly, encoding local descriptors is achieved by computing statistics of a background model, such as $k$-means, to embed each descriptor into a higher dimensional space, which are then aggregated. A popular encoding technique is VLAD [13], which aggregates first order statistics with sum pooling. The global representation is typically compared using the cosine distance.

In this work we investigate how VLAD encoding of CNN activations compares to the more recent triangulation embedding [14]. We further explore generalized max pooling [17], which balances the embeddings with regard to the final similarity score. To the best of our knowledge this has not yet been explored for $\mathrm{CNN}$ activations. Additionally, we evaluate the effect of PCA whitening on the different parts of the pipeline. Instead of using the cosine distance, we further explore exemplar classifiers to learn a similarity between representations.

\section{A. Feature Extraction}

Similar to our previous work [10], we use the activations from the penultimate layer from a trained CNN as features. The $\mathrm{CNN}$ is trained with four million raw (i.e. non-normalized) $32 \times 32$ patches randomly sampled from the script contour. The contours are obtained by a connected component analysis of the binarized images. We evaluate different network topologies to investigate their influence on the retrieval performance. The CNN activation features are then encoded to a global representation.

\section{B. Encoding}

Encoding consists of two steps, cf. Fig. 1: i) an embedding step where a, possible non-linear, function is applied to the local feature vectors in order to create a high dimensional representation, and ii) an aggregation step where the embedded local descriptors are pooled into a fixed-length vector. After a possible normalization step, the global representations can be compared to each other. In case of image retrieval this is commonly done by means of the cosine distance between the global representations.

1) Aggregation: Sum pooling is the standard aggregation method for pooling local descriptors. Max pooling requires embedding functions that associate a certain strength to one visual word which is not directly applicable to the most common embedding techniques that rely on higher order statistics [15]. The drawback of sum pooling is that it assumes that the descriptors in an image are independently and identically distributed (iid). More frequently occurring descriptors will be more influential in the final representation, a.k. a. visual burstiness. Thus, encoding methods have to normalize for this. In general, this is achieved during embedding or normalization. Alternatively, generalized max pooling (GMP) [17] and democratic aggregation [14] were proposed to counter this in the aggregation step. Both methods re-weight the patch statistics such that their influence regarding the final similarity score is equalized. Comparing both approaches [15], GMP showed superior performance. As indicated by its name, it generalizes max pooling by formulating the balancing problem of any embedding $\phi(\boldsymbol{x}) \in \mathbb{R}^{D}, \boldsymbol{x} \in \mathbb{R}^{D_{l}}$ as ridge regression problem. Therefore, let [15]

$$
\phi(\boldsymbol{x})^{\top} \boldsymbol{\xi}_{\mathrm{gmp}}(\mathcal{X})=C, \quad \forall \boldsymbol{x} \in \mathcal{X},
$$

where $\boldsymbol{x}$ is a local descriptor of the set of all local image descriptors $\mathcal{X}, \boldsymbol{\xi}_{\text {gmp }}$ is the GMP representation and $C$ is a constant which can be set arbitrarily since it has no influence when the representation is eventually $\ell_{2}$-normalized. Equ. (1) can be generalized for the $D \times n$ matrix $\Phi$ of all $n$ patch embeddings to

$$
\boldsymbol{\Phi}^{\top} \boldsymbol{\xi}_{\mathrm{gmp}}=\mathbf{1}_{n}
$$

where $\mathbf{1}_{n}$ denotes the vector of all constants set to 1 . This linear system can be turned into a least-squares ridge regression problem:

$$
\boldsymbol{\xi}_{\mathrm{gmp}}=\underset{\boldsymbol{\xi}}{\operatorname{argmin}}\left\|\boldsymbol{\Phi}^{\top} \boldsymbol{\xi}-\mathbf{1}_{n}\right\|^{2}+\lambda \|\left.\boldsymbol{\xi}\right|^{2}
$$

with $\lambda$ being a regularization term that stabilizes the solution. Equ. (3) can be computed using conjugate gradient descent (CGD). In the remainder, $\boldsymbol{\psi}$ denotes the aggregated result. CGD can typically be applied component-wise, i. e., on each $\phi_{k}$ (see below), to speed up the process. 
2) VLAD Embedding: VLAD can be viewed as a nonprobabilistic version of the Fisher Kernel [13] encoding only first order statistics. In combination with improvements such as whitening [18], intra-normalization [19], or residual normalization [20], VLAD is one of the standard encoding techniques. We successfully employed it for writer identification [21] and it has already been used in combination with deep-learning-based features for the task of classification and retrieval [22]-[24].

For VLAD encoding, the embedding function $\phi$ for the local descriptor $\boldsymbol{x}$ is computed by the residual to its nearest cluster center $\boldsymbol{\mu}_{k}$ of the dictionary $\mathcal{D}=\left\{\boldsymbol{\mu}_{k} \in \mathbb{R}^{D_{l}}, k=1, \ldots, K\right\}$ :

$$
\begin{aligned}
\phi_{\mathrm{VLAD}, k}(\boldsymbol{x}) & =\alpha_{k}(\boldsymbol{x})\left(\boldsymbol{x}-\boldsymbol{\mu}_{k}\right) \\
\alpha_{k}(\boldsymbol{x}) & =\left\{\begin{array}{ll}
1 & \text { if } k=\underset{j=1, \ldots, K}{\operatorname{argmin}}\left\|\boldsymbol{x}-\boldsymbol{\mu}_{j}\right\|_{2} \\
0 & \text { else }
\end{array} .\right.
\end{aligned}
$$

The full embedding follows as $\phi_{\mathrm{VLAD}}=\left(\phi_{1}^{\top}, \ldots, \phi_{K}^{\top}\right)^{\top}$.

VLAD encodings can be normalized to counter visual burstiness. The most popular normalization technique is power normalization, where each element $\psi_{i}$ of the aggregated embedding $\boldsymbol{\psi}$ is normalized as:

$$
\hat{\psi}_{i}=\operatorname{sign}\left(\psi_{i}\right)\left|\psi_{i}\right|^{p}, \forall \psi_{i} \in \boldsymbol{\psi},
$$

where $p$ is typically chosen to be 0.5 . This is also known as signed square root (SSR), which resembles the Hellinger kernel. Power normalization is typically followed by an $\ell_{2}$ normalization.

Other proposed normalization techniques are residual normalization [20] and intra normalization [19]. In the former one, each embedding $\phi_{k}$ is $\ell_{2}$-normalized. In intra normalization, the component-wise aggregated embeddings $\psi_{k}$ are $\ell_{2}$ normalized. It is also beneficial to decorrelate and whiten the representations to prevent an over-counting of co-occurrences [18]. This is commonly achieved by means of a principal component analysis with whitening (PCA whitening). PCA whitening can be applied either at the global representation $\psi$ or at the embeddings $\phi$ as well as their individual components $\psi_{k}$ and $\phi_{k}$, where the latter one was suggested by Delhumeau et al. [20] and referred to local coordinate systems (LCS).

3) Triangulation Embedding: Triangulation embedding [14] is very similar to VLAD with residual normalization. The normalized residuals of anchor points (= cluster centers) to feature descriptors are computed. These can be viewed as directions discarding absolute distances to cluster centers. The key difference is that no association function $(\alpha)$ is applied to the vectors. All normalized residuals are encoded, not just the nearest neighbor:

$$
\phi_{\mathrm{T}-\mathrm{Emb}, k}\left(\boldsymbol{x}_{t}\right)=\frac{\boldsymbol{x}_{t}-\boldsymbol{\mu}_{k}}{\left\|\boldsymbol{x}_{t}-\boldsymbol{\mu}_{k}\right\|_{2}} .
$$

Each embedding is whitened using PCA. However, instead of a dimensionality reduction, the authors propose to discard the largest $D_{l}$ eigenvalues and corresponding eigenvectors. This has the effect of reducing the variance of the cosine similarity between unrelated descriptors [14]. Each embedding is subsequently $\ell_{2}$ normalized and afterwards aggregated. Note, due to the $\ell_{2}$ normalization, the actual linear PCA whitening cannot be applied after the aggregation.

In contrast to power normalization, the authors [14] suggested the use of rotation normalization where the aggregated embedding is first rotated by means of a PCA and then powernormalized.

\section{Exemplar Support Vector Machines}

Recently, we proposed the use of Exemplar SVMs (ESVM) in the context of writer recognition [25]. Since the training and test sets of common writer recognition datasets are disjoint, an end-to-end classification of the writers is not possible. However, this allows to compute probe individual classifiers. The encoding of each probe sample serves as single positive instance while all samples from the training set are the negative samples for a linear support vector machine. This can be interpreted as computing an individual similarity for each query document. For evaluation, each other document is ranked according to the score of the probe ESVM.

\section{Evaluation}

\section{A. Datasets}

We evaluate the following publicly available datasets:

- The ICDAR13 dataset [26] consists of four samples per writer, where each writer contributed two samples in English and two samples in Greek. The test set consists of 250 writers and the training set contains 100 writers.

- The CVL dataset [27] (v. 1.1) contains 310 writers. 283 writers copied five texts (one in German and four in English). The remaining 27 writers contributed seven texts (one in German and six in English). We use the first five forms of all 310 writers as test set and all samples of the IAM dataset [28] for training.

- The KHATT dataset [29] consists of 1000 writers, each contributing four documents written in Arabic. The dataset is divided into three disjoint, i. e., writer independent, sets for training (70\%), validation (15\%) and testing (15\%).

The CVL and ICDAR13 datasets are text dependent, i.e., each writer copied the same four to seven forms. Conversely, two of the four forms of the KHATT dataset contain unique paragraphs. We binarized the IAM, CVL, and KHATT datasets using Otsu's method [30] to be more similar to the ICDAR13 dataset and making them more independent of the pen used.

The datasets are evaluated using a leave-one-sample-out cross validation. All results are given in terms of mean average precision (mAP), which computes the mean across the average precisions of all queries. The average precision of a query is computed by ranking the remaining documents according to their similarity to the query. Note all values are shown in percent.

\section{B. CNN Activation Features}

We tested several architectures. First, we employ a four layer (not counting pooling layers) deep CNN denoted as LeNet, which we used in our previous work [10]. It has two convolutional layers, each followed by a max pooling layer, 


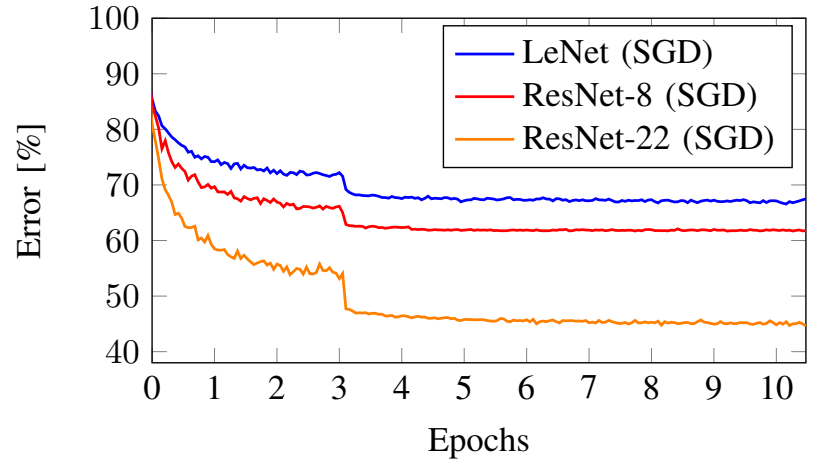

Fig. 2: Test error of the different architectures using the ICDAR13 training data (subset).

TABLE I: Evaluation of different decorrelation methods for CNN activation features extracted from the script contour (mAP, avg. of 5 runs, ICDAR13 test set)

\begin{tabular}{lccc}
\hline Method & Baseline & PCA wh. & ZCA wh. \\
\hline LeNet-A & 86.75 & 88.50 & 87.21 \\
LeNet-B & 88.02 & 88.58 & 89.21 \\
ResNet- 8 & 88.39 & 89.83 & 88.68 \\
ResNet-20 & 89.86 & $\mathbf{9 0 . 0 1}$ & 89.96 \\
\hline
\end{tabular}

and a fully connected layer followed by the classification layer.

In addition to the LeNet architecture, we evaluate two different ResNet models [31], [32] of different depths. We follow the architectural design and training procedure of He et al. [31] for the CIFAR10/100 datasets, where we evaluated eight and twenty layers deep CNNs, further denoted as ResNet-8 and ResNet-20.

The networks are optimized using SGD w.r.t. the crossentropy loss, a Nesterov momentum of 0.9 and a weight decay of $10^{-4}$. We use an initial learning rate of 0.01 which is multiplied by 0.1 after three epochs and once more after four epochs. The learning curves of the validation set $(20 \mathrm{k}$ independent patches) for the first ten epochs can be seen in Fig. 2.

Comparing the LeNet architecture with residual networks, the plot clearly shows the advantage of the latter. The deeper ResNet reduced the error rate below $50 \%$. This means in more than $50 \%$ a $32 \times 32$ patch can be assigned to the correct writer. However note that a deeper network also needs a longer training time.

\section{Decorrelation of CNN Activations}

Given the trained models, we can use them as feature extractors by forwarding the contour patches of a query sample through the networks. In all cases we used the 64-dimensional activations of the penultimate layer as features. They are subsequently $\ell_{2}$-normalized. For LeNet, we compared two different variants: LeNet-A refers to the results using the very same LeNet model of our previous work [10], i. e., the model after 20 epochs trained by a fixed learning rate of 0.01 , while LeNet-B denotes the results using the LeNet model after five
TABLE II: Comparison of VLAD in combination with LCS with triangulation embedding (avg. of 5 runs, ICDAR13 test set).

\begin{tabular}{lc}
\hline Method & mAP \\
\hline VLAD + ResNorm + LCS wh. & $\mathbf{9 0 . 4 4}$ \\
VLAD + ResNorm + LCS++ & 90.42 \\
T-Emb & 89.71 \\
T-Emb16 & 87.65 \\
\hline
\end{tabular}

epochs using the proposed learning rate schedule. We also used the models after the fifth epoch for the ResNet models since the error stagnated at this point. Additionally, we evaluated the effect of decorrelating the activations by means of i) PCA rotation and whitening and ii) zero component analysis (ZCA) with whitening. ZCA whitening rotates the data back to be as close to the input as possible. In the field of deep learning, ZCA whitening is more common than PCA whitening.

For the baseline encoding, we employ VLAD encoding (a pure sum pooling, i.e., no embedding achieves $78.80 \mathrm{mAP}$ ). Since VLAD encoding depends much on a good background model, we compute five different VLAD-encodings and give the average result. The background model is computed by a mini-batch version of $k$-means [33] using different seeds and different selection of training samples. In this way, we have a large variability in the background models. The VLAD encodings are individually normalized by power normalization $(p=0.5)$ followed by an $\ell_{2}$ normalization.

Tab. I depicts all mAP results. The baseline results (first column) show that LeNet-A is inferior to LeNet-B, suggesting that a learning rate schedule is beneficial for the final feature representation. Any LeNet configuration performs worse than ResNets. A decorrelation is especially beneficial for the smaller networks. However, no decorrelation method is significantly superior. Interestingly, a PCA whitening of the ResNet-20 model achieves the highest results. Exchanging SSR with intra normalization [19] or residual normalization [20] for the baseline experiment performed worse with 89.44 and 88.31 $\mathrm{mAP}$, respectively (no normalization at all: $88.46 \mathrm{mAP}$ ). In the following experiments, we used the activation features of the ResNet-20 model, if not mentioned otherwise in its non-whitened version.

\section{From VLAD to Triangulation Embedding}

Since triangulation embedding is an adapted VLAD encoding, it is interesting to explore the individual adjustments. Instead of T-emb's global PCA whitening, we use LCS [20] with whitening on $\ell_{2}$ normalized residuals (VLAD + ResNorm + LCS wh.). This is compared with a variant where the first component of each PCA transformed $\phi_{k}$ is discarded $(\mathrm{VLAD}+\mathrm{ResNorm}+\mathrm{LCS}++)$. Two variants of triangulation embedding are used for comparison. The first one uses 100 cluster centers similar to VLAD (T-Emb), and the other one uses 16 components (T-Emb16). The latter is the default value of triangulation embedding [14] to speed up the aggregation process. Tab. II shows that VLAD in combination with LCS whitening is superior to triangulation embedding. This suggests that the power of triangulation is not based on the aggregation 
TABLE III: Evaluation of CNN activation features in combination with T-Emb (mAP, avg. of 5 runs, ICDAR13 test set).

\begin{tabular}{lccc}
\hline Method & VLAD++ & T-Emb & T-Emb16 \\
\hline Baseline & $\mathbf{9 0 . 4 4}$ & 89.71 & 87.65 \\
RotNorm & 81.73 & 88.78 & 88.81 \\
PCA wh. & 82.23 & 89.39 & 89.60 \\
joint PCA wh. & 83.04 & 90.08 & 90.21 \\
\hline
\end{tabular}

TABLE IV: Comparison of sum pooling and GMP (mAP, avg. of 5 runs, ICDAR13 test set).

\begin{tabular}{lccc}
\hline Method & Sum & $\mathrm{GMP}_{\lambda=1}$ & $\mathrm{GMP}_{\lambda=10^{3}}$ \\
\hline VLAD++ & 90.44 & 89.74 & $\mathbf{9 0 . 6 6}$ \\
T-Emb16 & 87.65 & 78.59 & $\mathbf{8 9 . 1 1}$ \\
\hline
\end{tabular}

of more descriptors but instead on the positive effect of PCA whitening.

To make the evaluation process completely fair, we also evaluated the effect of rotation normalization (RotNorm), which was proposed in combination with triangulation embedding [14]. Additionally, we evaluated a simple PCA whitening (PCA wh.) and a joint PCA whitening (joint PCA wh.), where the encodings of the five runs are concatenated and jointly whitened. Tab. III shows that another decorrelation of the final aggregated embedding is not beneficial. While a joint PCA whitening shows some improvements for triangulation embedding, it worsens the results of VLAD plus residual normalization and LCS whitening (VLAD++).

\section{E. Sum Pooling vs. Generalized Max Pooling}

The use of GMP requires the proper choice of the regularization parameter $\lambda$. Murray et al. [15] suggested that a factor of 1.0 works well in practice. However, cross-validating its effect on the ICDAR13 training set showed that a much higher value of $\lambda=1000$ is preferable among the embeddings.

In Tab. IV, we compare sum pooling with GMP using the two embeddings VLAD++ and T-Emb with 16 components (T-Emb16). When using a proper regularization parameter, GMP outperforms sum pooling by a small factor. However, the benefit is small and another parameter $(\lambda)$ needs to be cross-validated.

\section{F. Exemplar Support Vector Machines and PCA whitening}

We compare the effectiveness of ESVMs with: VLAD plus SSR (VLAD), VLAD with LCS whitening (VLAD++) and triangulation embedding (T-Emb16). All methods use GMP $(\lambda=1000)$, the margin parameter of the SVM is for each method cross-validated using the training set. Comparing the different results of Tab. Va, we see that all methods perform well. Interestingly, the basic VLAD method performs the best. Hence, we can conclude that the additional decorrelation with PCA, which is part of LCS and triangulation embedding does not necessarily improve the recognition results. Additionally, we investigated the effect of the PCA whitened version of the CNN activation features. Tab. Vb shows that this step improves the recognition of the basic VLAD method and triangulation embedding.
TABLE V: Evaluation of ESVMs using (a) different embeddings, aggregated with GMP and (b) additional PCA whitening of the CNN activation features (mAP, avg. of 5 runs, ICDAR13 test set).

\begin{tabular}{lcc}
\hline Method & Baseline & ESVM \\
\hline VLAD & 89.86 & $\mathbf{9 1 . 7 2}$ \\
VLAD++ & 90.66 & 91.64 \\
T-Emb16 & 89.11 & 91.56 \\
\hline
\end{tabular}

(a) $\mathrm{CNN}-\mathrm{AF}$

\begin{tabular}{cc}
\hline Baseline & ESVM \\
\hline 90.19 & $\mathbf{9 3 . 2 4}$ \\
90.41 & 91.53 \\
89.24 & 92.73 \\
\hline
\end{tabular}

(b) $\mathrm{CNN}-\mathrm{AF}+\mathrm{PCA}$ wh.

TABLE VI: Comparison with the state of the art.

\begin{tabular}{lcccccc}
\hline Method & Top-1 & H-2 & H-3 & S-5 & S-10 & mAP \\
\hline$[12]$ & 99.0 & 84.4 & 68.1 & 99.2 & 99.6 & - \\
{$[25]$} & $\mathbf{9 9 . 7}$ & 84.8 & 63.5 & $\mathbf{9 9 . 8}$ & 99.8 & 89.4 \\
\hline VLAD & 99.0 & 85.3 & 68.6 & 99.4 & 99.7 & 90.2 \\
VLAD + E & 99.6 & $\mathbf{8 9 . 8}$ & $\mathbf{7 7 . 0}$ & $\mathbf{9 9 . 8}$ & $\mathbf{9 9 . 9}$ & $\mathbf{9 3 . 2}$ \\
\hline
\end{tabular}

(a) ICDAR13

\begin{tabular}{lcccccc}
\hline Method & Top-1 & H-2 & H-3 & S-5 & S-10 & mAP \\
\hline$[12]$ & $\mathbf{9 9 . 7}$ & $\mathbf{9 9 . 0}$ & $\mathbf{9 7 . 9}$ & $\mathbf{9 9 . 8}$ & $\mathbf{1 0 0}$ & - \\
{$[25]$} & 99.2 & 98.4 & 97.1 & 99.6 & 99.7 & 98.0 \\
\hline VLAD & 99.2 & 98.4 & 96.1 & 99.5 & 99.6 & 97.4 \\
VLAD + E & 99.5 & $\mathbf{9 9 . 0}$ & 97.7 & 99.6 & 99.8 & $\mathbf{9 8 . 4}$ \\
\hline
\end{tabular}

(b) CVL

\begin{tabular}{lcccccc}
\hline Method & Top-1 & H-2 & H-3 & S-5 & S-10 & mAP \\
\hline$[25]$ & 99.5 & 96.5 & 92.5 & 99.5 & 99.5 & 97.2 \\
\hline VLAD & 99.1 & 93.8 & 88.2 & 99.4 & 99.6 & 95.5 \\
VLAD + E & $\mathbf{9 9 . 6}$ & $\mathbf{9 7 . 6}$ & $\mathbf{9 4 . 5}$ & $\mathbf{9 9 . 7}$ & $\mathbf{9 9 . 7}$ & $\mathbf{9 8 . 0}$ \\
\hline
\end{tabular}

(c) KHATT

\section{G. Comparison with the State of the Art}

Finally, we compare the results with the state of the art on the ICDAR13, CVL, and KHATT datasets. Additional to the mAP, we depict the following common writer identification/retrieval measures: Top-1 gives the probability that the first retrieved result stems from the same writer. While Soft-5 (S-5)/Soft-10 (S-10) requires that one of the top five/ten results is written by the query writer, Hard-2 (H-2)/Hard-3 (H-3) requires that all two/three top ranked results are from the same writer.

We used VLAD in combination with generalized max pooling $(\lambda=1000)$ and SSR. Additionally, we show the results with the Exemplar SVM step. We compare our results with our previous work [25] and the work of Tan and Wu [12]. To the best of our knowledge, these two methods are currently leading the ICDAR13, CVL and KHATT datasets.

Tab. VI shows all results. Our method achieves results similar to the state of the art on all datasets while even being superior on the ICDAR13 and KHATT dataset. Previous evaluations on the CVL dataset [25] suggest that a better background model could further improve the results.

\section{CONCLUSion}

In this work, we evaluated several effects related to the encoding of local descriptors. We showed that 1) a deeper CNN only slightly achieves higher retrieval performance 2) 
triangulation embedding is not better than VLAD when either the local descriptors or the embeddings are decorrelated by means of a PCA whitening. 3) Generalized max pooling is slightly better than sum pooling but is sensitive to a proper regularization. 4) When using Exemplar SVMs, all three methods perform similarly well, where VLAD in combination with power normalization performs slightly better. The proposed combination of deep CNN activation features, VLAD encoding, normalization and Exemplar SVMs is similar or better than state-of-the-art methods on three publicly available datasets. For future work, we would like to explore different layers than the penultimate layer for feature extraction. Furthermore, larger input patch sizes could also be beneficial.

\section{REFERENCES}

[1] A. Brink, J. Smit, M. Bulacu, and L. Schomaker, "Writer Identification Using Directional Ink-Trace Width Measurements," Pattern Recognition, vol. 45, no. 1, pp. 162-171, 2012. 1

[2] T. Gilliam, R. C. Wilson, and J. A. Clark, "Scribe Identification in Medieval English Manuscripts," in 20th International Conference on Pattern Recognition (ICPR), 2010, pp. 1880-1883. 1

[3] D. Fecker, A. Asit, V. Margner, J. El-Sana, T. Fingscheidt, V. Märgner, J. El-Sana, and T. Fingscheidt, "Writer Identification for Historical Arabic Documents," in 2014 22nd International Conference on Pattern Recognition (ICPR), 2014, pp. 3050-3055. 1

[4] S. He and L. Schomaker, "Delta-n Hinge: Rotation-Invariant Features for Writer Identification," in 2014 22nd International Conference on Pattern Recognition (ICPR), 2014, pp. 2023-2028. 1

[5] A. Krizhevsky, I. Sutskever, and G. E. Hinton, "ImageNet Classification with Deep Convolutional Neural Networks," in Advances In Neural Information Processing Systems 25. Curran Associates, Inc., 2012, pp. 1097-1105. 1

[6] T. Bluche, H. Ney, and C. Kermorvant, "Feature Extraction with Convolutional Neural Networks for Handwritten Word Recognition," in 2013 12th International Conference on Document Analysis and Recognition, 2013, pp. 285-289. 1

[7] M. Jaderberg, A. Vedaldi, and A. Zisserman, "Deep Features for Text Spotting," in Computer Vision - ECCV 2014. Springer International Publishing, 2014, vol. 8692, pp. 512-528. 1

[8] F. Wahlberg, T. Wilkinson, and A. Brun, "Historical Manuscript Production Date Estimation Using Deep Convolutional Neural Networks," in 2016 15th International Conference on Frontiers in Handwriting Recognition (ICFHR), 2016, pp. 205-210. 1

[9] S. Fiel and R. Sablatnig, "Writer Identification and Retrieval Using a Convolutional Neural Network," in Computer Analysis of Images and Patterns: 16th International Conference, CAIP 2015, Valletta, Malta, September 2-4, 2015, Proceedings, Part II, G. Azzopardi and N. Petkov, Eds. Springer International Publishing, 2015, pp. 26-37. 1

[10] V. Christlein, D. Bernecker, A. Maier, and E. Angelopoulou, "Offline Writer Identification Using Convolutional Neural Network Activation Features," in Pattern Recognition: 37th German Conference, GCPR 2015, Aachen, Germany, October 7-10, 2015, Proceedings, J. Gall, P. Gehler, and B. Leibe, Eds. Springer International Publishing, 2015, vol. 9358, pp. 540-552. 1, 2, 3, 4

[11] L. Xing and Y. Qiao, "DeepWriter: A Multi-Stream Deep CNN for Textindependent Writer Identification," in 2016 15th International Conference on Frontiers in Handwriting Recognition (ICFHR), 2016, pp. 584-589. 1

[12] Y. Tang and X. Wu, "Text-Independent Writer Identification via CNN Features and Joint Bayesian," in 2016 15th International Conference on Frontiers in Handwriting Recognition (ICFHR), 2016, pp. 566-571. 1, 5

[13] H. Jégou, F. Perronnin, M. Douze, J. Sánchez, P. Pérez, and C. Schmid, "Aggregating Local Image Descriptors into Compact Codes," IEEE Transactions on Pattern Analysis and Machine Intelligence, vol. 34, no. 9, pp. 1704-1716, 2012. 1, 2, 3

[14] H. Jégou and A. Zisserman, "Triangulation Embedding and Democratic Aggregation for Image Search," in 2014 IEEE Conference on Computer Vision and Pattern Recognition (CVPR), 2014, pp. 3310-3317. 1, 2, 3, 4,5
[15] N. Murray, H. Jegou, F. Perronnin, and A. Zisserman, "Interferences in Match Kernels," IEEE Transactions on Pattern Analysis and Machine Intelligence, vol. PP, no. 99, p. 1, 2016. 1, 2, 5

[16] T. Hoang, T.-T. Do, D.-K. L. Tan, and N.-M. Cheung, "Selective Deep Convolutional Features for Image Retrieval," in ACM Multimedia conference, 2017. 1

[17] N. Murray and F. Perronnin, "Generalized Max Pooling," in 2014 IEEE Conference on Computer Vision and Pattern Recognition, 2014, pp. 2473-2480. 1, 2

[18] H. Jégou and C. Ondřej, "Negative Evidences and Co-occurences in Image Retrieval: The Benefit of PCA and Whitening," in Computer Vision - ECCV 2012: 12th European Conference on Computer Vision, Florence, Italy, October 7-13, 2012, Proceedings, Part II, A. Fitzgibbon, S. Lazebnik, P. Perona, Y. Sato, and C. Schmid, Eds. Springer Berlin Heidelberg, 2012, pp. 774-787. 3

[19] R. Arandjelovic and A. Zisserman, "All About VLAD," in 2013 IEEE Conference on Computer Vision and Pattern Recognition (CVPR), 2013, pp. $1578-1585.3,4$

[20] J. Delhumeau, P.-H. Gosselin, H. Jégou, and P. Pérez, "Revisiting the VLAD Image Representation," in 21st ACM International Conference on Multimedia - MM '13. ACM, 2013, pp. 653-656. 3, 4

[21] V. Christlein, D. Bernecker, and E. Angelopoulou, "Writer Identification using VLAD Encoded Contour-Zernike Moments," in 2015 13th International Conference on Document Analysis and Recognition (ICDAR), 2015, pp. 906-910. 3

[22] Y. Gong, L. Wang, R. Guo, and S. Lazebnik, "Multi-scale Orderless Pooling of Deep Convolutional Activation Features," in Computer Vision - ECCV 2014, D. Fleet, T. Pajdla, B. Schiele, and T. Tuytelaars, Eds. Springer International Publishing, 2014, vol. 8695, pp. 392-407. 3

[23] J. Y. H. Ng, F. Yang, and L. S. Davis, "Exploiting Local Features from Deep Networks for Image Retrieval," in 2015 IEEE Computer Society Conference on Computer Vision and Pattern Recognition Workshops (CVPRW), 2015, pp. 53-61. 3

[24] M. Paulin, J. Mairal, M. Douze, Z. Harchaoui, F. Perronnin, and C. Schmid, "Convolutional Patch Representations for Image Retrieval: An Unsupervised Approach," International Journal of Computer Vision, vol. 121, no. 1, pp. 149-168, 2016. 3

[25] V. Christlein, D. Bernecker, F. Hönig, A. Maier, and E. Angelopoulou, "Writer Identification Using GMM Supervectors and Exemplar-SVMs," Pattern Recognition, vol. 63, pp. 258-267, 2017. 3, 5

[26] G. Louloudis, B. Gatos, N. Stamatopoulos, and A. Papandreou, "ICDAR 2013 Competition on Writer Identification," in 2013 12th International Conference on Document Analysis and Recognition (ICDAR), 2013, pp. 1397-1401. 3

[27] F. Kleber, S. Fiel, M. M. Diem, and R. Sablatnig, "CVL-DataBase: An Off-Line Database for Writer Retrieval, Writer Identification and Word Spotting," in 2013 12th International Conference on Document Analysis and Recognition (ICDAR), 2013, pp. 560 - 564. 3

[28] U.-V. Marti and H. Bunke, "The IAM-Database: An English Sentence Database for Offline Handwriting Recognition," International Journal on Document Analysis and Recognition, vol. 5, pp. 39-46, 2002. 3

[29] S. A. Mahmoud, I. Ahmad, W. G. Al-Khatib, M. Alshayeb, M. Tanvir Parvez, V. Märgner, and G. A. Fink, "KHATT: An Open Arabic Offline Handwritten Text Database," Pattern Recognition, vol. 47, no. 3, pp. 1096-1112, 2014. 3

[30] N. Otsu, "A Threshold Selection Method from Gray-Level Histograms," Systems, Man, and Cybernetics, IEEE Transactions on, vol. 9, no. 1, pp. $62-66,1979.3$

[31] K. He, X. Zhang, S. Ren, and J. Sun, "Deep Residual Learning for Image Recognition," in 2016 IEEE Conference on Computer Vision and Pattern Recognition (CVPR), 2016, pp. 770-778. 4

[32] — , "Identity Mappings in Deep Residual Networks," in Computer Vision - ECCV 2016: 14th European Conference, Amsterdam, The Netherlands, October 11-14, 2016, Proceedings, Part IV, B. Leibe, J. Matas, N. Sebe, and M. Welling, Eds. Springer International Publishing, 2016, pp. 630-645. 4

[33] D. Sculley, "Web-Scale K-means Clustering," in 19th International Conference on World Wide Web, ser. WWW '10. ACM, 2010, pp. 1177-1178. 4 